\title{
DESENVOLVIMENTO, VALIDAÇÃO E APLICAÇÃO DE UM INSTRUMENTO DE CARACTERIZAÇÃO DAS FERIDAS
}

\section{DEVELOPMENT, VALIDATION AND APPLICATION OF A TOOL TO MEASURE WOUND CHARACTERISTICS}

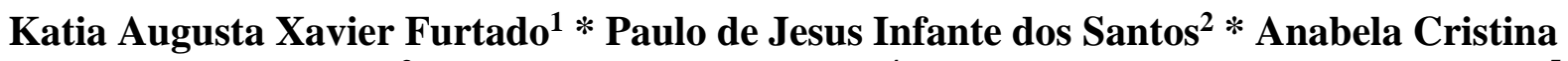 \\ Cavaco Ferreira Afonso ${ }^{3}$ * Filipe José Paulo Gomes ${ }^{*}$. José Carlos Casaquinha Carranca 5 * \\ Manuel José Lopes ${ }^{6}$
}

\begin{abstract}
RESUMO
Introdução: A prevalência das feridas é de grande variabilidade entre países, sobretudo condicionada por estudos de características muito heterogéneas. A presente investigação foi efetuada nas unidades de internamento da Rede Nacional de Cuidados Continuados do Alentejo (RNCCI). Objetivo: Desenvolver e validar um instrumento clínico útil na medição das características das feridas. Método: Estudo desenvolvido em quatro fases: (1) Construção do instrumento; (2) Reformulação do instrumento com discussão num pequeno grupo de enfermeiros da prática clínica para melhorar viabilidade e relevância clínica; (3) Revisão da semântica; (4) Avaliação da confiabilidade entre observadores. Resultados: O instrumento final é constituído por quatro partes. A primeira recolhe variáveis sociodemográficas. A segunda parte inclui as patologias associadas. A terceira recolhe informação sobre a prevalência das diferentes tipologias de feridas. A quarta e última parte do instrumento recolhe informação sobre o tempo de enfermagem dependido na realização do penso e frequência. Conclusão: Este instrumento, agora disponível, permite identificar o número e tipologia das feridas, caracterizar a gravidade, a localização anatómica, os cuidados associados ao tratamento e ainda identificar os antecedentes relevantes do doente.

Plavras-chave: Estudos Transversais; Estudos Epidemiológicos; Feridas; Assistência de Longa Duração; Casa de Repouso; Úlcera por Pressão.
\end{abstract}

\begin{abstract}
Introduction: Wounds prevalence vary enormously along countries, usually because studies conducted have heterogeneous characteristics. The present research took place in long term units in Alentejo, Portugal. Aim: The aim of this study is to develop and validate a clinical useful instrument to measure wounds characteristics. Methods: This study was conducted in four stages: (1) Development of the instrument to measure wounds prevalence based on literature review; (2) In a second step, the form was discussed in a focus group interviews with experienced nurses to enhance clinical relevance and usability. (3) Semantic revision based on the answers from the focus group about descriptions and definitions and whether the form was simple and clear to use; (4) A preliminary statistical evaluation (item discrimination and interrater reliability) was conducted. Results: The final instrument has four sections. The first one collects patient background information. The second one, information about diseases present. The third one focus on prevalence of different wounds typology. The last one, is dedicated to nursing time spent during dressing changes. Conclusion: the instrument now available allows to identify number and typology of wounds, describe their grades, location, nursing care demanded and identifies relevant pathologies associated.

Keyword: Cross-Sectional Studies; Epidemiologic Studies; Wounds; Long Term Care; Nursing Homes; Pressure Ulcer.

\footnotetext{
${ }^{1}$ Enfermeira Especialista em Saúde Comunitária, Unidade Local de Saúde do Norte Alentejano. Consulta externa. Doutoranda em Enfermagem. Orcid: https://orcid.org/0000-0002-1130-3678. E-mail: kaxfurtado@gmail.com

${ }_{2}^{2}$ Professor Doutor. Universidade de Évora. Departamento de Matemática. Orcid: https://orcid.org/0000-0002-1644-9502

${ }^{3}$ Professora Doutora. Universidade de Évora. Departamento de Matemática. Orcid: https://orcid.org/0000-0002-5517-4855

4 Enfermeiro Especialista em Saúde Comunitária, Centro de Saúde da Covilhã. Mestre em cuidados Paliativos. Orcid: https://orcid.org/0000-0002-1207-6294

5 Enfermeiro Especialista em Saúde Comunitária, ELCOS Sociedade Portuguesa de Feridas. Orcid: https://orcid.org/0000-00020290-6127

${ }^{6}$ Professor Doutor. Universidade de Évora. Departamento de Enfermagem. Orcid: https://orcid.org/0000-0002-7554-8041
} 
INTRODUÇÃO

A Rede Nacional de Cuidados Continuados Integrados (RNCCI) constituiu uma inovação organizacional, tendo sido criada com o desígnio de responder às novas necessidades de saúde e sociais dos portugueses. Sendo o tratamento das feridas um dos principais motivos de ingresso nestas unidades, muitos utentes nunca chegam a cicatrizar as suas feridas.

As feridas de difícil cicatrização constituem um grave problema de saúde pública. Elas correspondem a uma perda anatómica e fisiológica da continuidade da pele, traduzindo uma problemática com elevado impacto na nossa sociedade, nomeadamente no que respeita a custos tangíveis (com impacto direto na economia), e a outros não tangíveis como a baixa qualidade de vida ou a elevada morbilidade ${ }^{1-3}$.

Embora esteja disponível informação variada acerca da prevalência das feridas, do número de doentes portador, num determinado período, estes dados, de per si, não são suficientes para avaliar o impacto dos processos de cuidados nos resultados clínicos obtidos. Colocam-se-nos ainda várias questões determinantes para esta avaliação, sendo importante conhecer o tempo de cicatrização das feridas, a sua localização anatómica, qual a situação clínica de base, do doente; qual a gravidade da situação clínica, quais os cuidados de enfermagem associados aos resultados obtidos?
Estes dados, no seu conjunto, constituem uma excelente ferramenta para a compreensão e melhoria da gestão do processo de cuidados à pessoa com feridas. $\mathrm{A}$ sua compreensão é de importância decisiva pois como sabemos (perante uma incidência estável e com tempos de cicatrização progressivamente menores), é possível reduzir a prevalência.

O conhecimento dos dados acima indicados revela-se ainda fundamental para planificação de uma atuação a montante, para que os processos de cuidados possam atuar sobre as causas, inibindo-se assim, por um lado, os fatores predisponentes (que reduzirão a incidência) e, por outro, diminuindo-se os tempos de cicatrização (reduzindo a prevalência).

Embora a prevalência de feridas se manifeste em crescendo (nas úlceras por pressão, nas úlceras de perna e nas feridas diabéticas), até à data, não existem instrumentos validados que meçam as diferentes tipologias de feridas: os seus fatores de risco e as circunstâncias adversas a elas associadas, a sua localização, o tempo despendido na realização do penso - a história da ferida do doente. Apenas os instrumentos de prevalência de úlceras por pressão (UPP) têm sido utilizados em grande extensão ${ }^{4}$.

$\mathrm{O}$ instrumento que agora se propõe validar é de particular interesse e impacto na comunidade clínica, porquanto o envelhecimento demográfico nos países desenvolvidos tem vindo a aumentar 5, 
associando-se e esse envelhecimento uma evidente tendência para um aumento de incidência e de prevalência das complicações de saúde, particularmente expressiva em alguns países ${ }^{5,6}$.

Ora as alterações da pele são as que mais refletem os efeitos do envelhecimento, sendo as mais visíveis a lenta renovação celular, a redução da função barreira com a perda da rede vascular e glandular, da hidratação celular, do tecido fibroso, com a perda de elasticidade e de resistência - o que gera elevadas complicações e morbilidade associadas, aumentando o risco de desenvolvimento de feridas, nomeadamente de descolamentos de pele e de úlceras por pressão ${ }^{7,8}$. Adicionalmente, quer devido à presença de comorbilidade, de determinados estilos de vida e de outros determinantes sociais da saúde, verifica-se um aumento do tempo de cicatrização. Mas, pior: verifica-se um aumento do risco de viver durante toda a vida, com uma ou mais feridas.

Observa-se assim que a prevalência das feridas é de grande variabilidade entre países, sendo os resultados condicionados por estudos de características muito heterogéneas 9. A maior parte dos estudos é de âmbito regional ou institucional, sem uniformidade quanto à metodologia ou recolha de dados ${ }^{4,10}$. Por outro lado, os dados existentes referem-se quase sempre a feridas de difícil cicatrização, assumindo-se que a sua prevalência global será entre 1 e $2 \%$ para a maioria dos países desenvolvidos ${ }^{3}$. As feridas de difícil cicatrização incluem: úlceras por pressão, úlceras de perna, úlceras de origem diabética, feridas malignas e queimaduras.

Situando-nos na realidade portuguesa, observa-se que ela coincide com o contexto internacional, tendo vindo a ser descrita com estudos setoriais, adstritos a algumas regiões ou instituições e com grande variação em termos de metodologias utilizadas. O primeiro estudo deste género no nosso país, que reporta a um conjunto de centros de saúde da região de Lisboa, concluiu que a prevalência de úlcera de perna, avaliada num período de duas semanas, foi de $1,41 \%$, sendo $80 \%$ de origem venosa, $5 \%$ arteriais e $15 \%$ de origem mista ${ }^{11}$. Surgiram, mais tarde, outros estudos, nomeadamente 12 , relativo à população assistida nos centros de saúde de Leiria, onde se destaca uma prevalência de feridas crónicas de $0,84 \%$, sendo mais frequentes as de causa vascular (36\%), das quais $78 \%$ seriam de origem venosa.

Mantendo-nos no envelhecimento demográfico e na morbilidade consequente, forcar-nos-emos na população institucionalizada da Rede Nacional de Cuidados Continuados Integrados do Alentejo (RNCCI), uma intervenção conjunta da Saúde e do Apoio Social, conforme Decreto-Lei n. ${ }^{\circ}$ 101/2006, de 6 de junho. As Unidades de Cuidados Continuados Integrados (UCCI) são constituídas por unidades de ambulatório e unidades de internamento pretendendo já dar resposta ao envelhecimento demográfico e seu impacto, referido pela OCDE. Estas 
últimas incluem três tipologias com objetivos distintos: Unidades de Convalescença (UC), Unidades de Média Duração e Reabilitação (UMDR) e Unidades de Longa Duração e Manutenção (ULDM). As Unidades de Cuidados Paliativos (UCP), outrora pertencentes à RNCCI, foram entretanto dissociadas desta última, devido à criação da Rede Nacional de Cuidados Paliativos 13

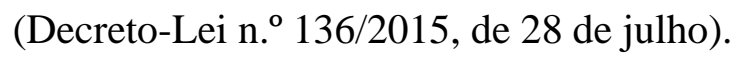

Em relação à caracterização dos utentes, a população da RNCCI é constituída predominantemente por pessoas com idade superior a 65 anos $(83,2 \%$ em 2020). De entre estas, a população com idade superior a 80 anos representou $50,9 \%$ do total, $\left(51,9 \%\right.$ no $1^{\circ}$ semestre de 2019), com maior prevalência do sexo feminino $(55 \%){ }^{14}$. Em relação a diagnósticos associados, prevalece a patologia vascular cerebral $(24,8 \%)$, fratura de colo do fémur $(12 \%)$ e úlcera crónica da pele $(9,4 \%)$ 14.

Todavia, o grande motivo de referenciação para a RNCCI é a dependência na realização de atividades de vida diária $(90,4 \%)$, estando a necessidade de tratamento de feridas / úlceras de pressão presente em $37 \%$ dos utentes referenciados ${ }^{14}$. Dados recentes ${ }^{14}$ mostram que a incidência de úlceras por pressão, na população assistida/institucionalizada na RNCCI, foi de $3,7 \%$ e que a prevalência de UPP foi de $16,4 \%$.

O tempo de cicatrização das UPP é elevado, observando-se que a maioria dos utentes com tempos de evolução superiores a um ano se encontra em Unidades de Convalescença (UC) e Unidades de Média Duração e Reabilitação (UMDR), respetivamente $2,5 \%$ e $9,9 \%$.

Em face da realidade identificada, é indispensável conhecer melhor os fatores de risco associados ao desenvolvimento das feridas e os métodos mais apropriados para reduzir a sua incidência e tempo de cicatrização.

$\mathrm{O}$ estudo que realizamos tem como objetivo criar um instrumento capaz de caracterizar a gravidade das feridas, identificar a sua localização anatómica e os antecedentes clínicos relevantes do doente, de modo a facilitar a realização de um diagnóstico para posterior intervenção.

Pensamos que este instrumento, até ao momento inexistente em Portugal, pela informação que acrescenta e padronização de recolha de dados que institui, virá aumentar o conhecimento acerca da realidade dos doentes com ferida e promover, para eles, ganhos em saúde e qualidade de vida.

Seguindo o pensamento 10,15,16, permitirá ainda desenvolver, de forma estimulante, atividades de benchmarking institucional dos projetos de qualidade realizados com sucesso nos cidadãos institucionalizados nas UCCI e em outras instituições do país. 


\section{MÉTODO}

\section{Desenho do estudo}

Trata-se de um estudo de desenvolvimento e validação de um instrumento de caraterização de feridas, em quatro fases: (1) Construção do instrumento, baseado no levantamento da literatura e opinião de peritos; (2) Reformulação do instrumento com discussão num pequeno grupo de enfermeiros da prática clínica para melhorar a viabilidade e relevância clínicapainel Delphi; (3) Revisão da semântica do instrumento; (4) Avaliação da confiabilidade entre observadores.

\section{Fase 1 - Construção do instrumento}

Foram extraídos componentes importantes dos instrumentos existentes avaliados em revisões sistemáticas ou metaanálises ${ }^{17}$ e os requisitos internacionais ${ }^{10}$, para obter informação das seguintes áreas: características sociodemográficas dos doentes; fatores de risco e circunstâncias adversas; tipologia das feridas, localização anatómica e tempo de duração das mesmas; tempo e frequência de realização do penso à ferida mais complicada. Um grupo de 5 peritos da Sociedade Portuguesa de Feridas (ELCOS), 3 enfermeiros especialistas com formação avançada em feridas com mais de 10 anos de experiência, 1 cirurgião geral e 1 enfermeiro doutorado em engenharia multimédia, construíram o protótipo do instrumento, com base nos critérios e áreas anteriormente referenciadas.

\section{Fase 2 - Validade de conteúdo}

Esta fase refere-se ao grau em que o conteúdo de um instrumento reflete adequadamente o construto que está sendo medido. A validade do conteúdo representa o esforço inicial para melhorar a validação do construto de um instrumento ${ }^{18}$. Após a elaboração do instrumento, seguiu-se a discussão com 10 enfermeiros da prática clínica para aumentar a relevância clínica e utilidade do mesmo. Os enfermeiros foram selecionados por conveniência, sendo todos coordenadores de grupos de prevenção e tratamento de feridas em diferentes instituições. Em cada reunião foi pedido que os enfermeiros aplicassem a nova versão do instrumento a 10 doentes da sua instituição e fizessem críticas ao mesmo. Foram realizadas 3 reuniões virtuais, altura em que ficaram esgotadas as sugestões de melhoria. As sugestões de melhoria, aprovadas por unanimidade ou maioria, foram incluídas na versão subsequente do instrumento. Os restantes itens foram revistos ou eliminados.

\section{Fase 3 - Semântica do Instrumento}

Foi feito um manual de instruções sobre o significado de cada item avaliado no instrumento, disponível em www.sociedadeferidas.pt. Todas as questões são de escolha múltipla, exceto a idade, o local de residência e o tempo de realização do penso à ferida mais grave, para facilitar o seu preenchimento. A primeira parte do instrumento recolhe dados 
sociodemográficos, como a idade, o género, o estado civil, o tipo de família classificadas de acordo com o International Classification for Nursing Practice (ICNP, versão 2018) e o local de residência. As patologias e circunstâncias adversas avaliadas foram posteriormente agrupados nos 19 grupos de doenças definidas no International Classification of Diseases (ICD-10) da Organização Mundial de Saúde. Já as diferentes tipologias de feridas foram classificadas de acordo com as definidas pelo ICNP. As úlceras por pressão e lesões por humidade foram classificadas de acordo com a European Pressure Ulcer Advisory Panel ${ }^{19}$. As definições de ferida cirúrgica, fístula e abcesso foram retiradas European Centre for Disease Prevention and Control.

\section{Fase 4 - Confiabilidade}

A confiabilidade do instrumento, isto é a capacidade em reproduzir um resultado de forma consistente no tempo e no espaço, ou a partir de dois observadores, constitui um dos principais critérios de avaliação da qualidade dos instrumentos ${ }^{20}$. Foram selecionadas duas instituições da RNCCI onde dois enfermeiros observaram uma amostra por conveniência de 16 doentes. Cada um preencheu um instrumento por doente e de forma independente.

\section{Local e período do estudo}

A presente investigação foi efetuada nas unidades de internamento da rede de cuidados continuados do Alentejo, abrangendo as UCCI dos distritos de Alentejo Litoral, Portalegre, Évora e Beja. A Região do Alentejo, situada no sul de Portugal, é caracterizada pela sua grande área geográfica, por uma população maioritariamente com dificuldades socioeconómicas, envelhecida e com várias morbilidades ${ }^{6,14}$. Este estudo inclui ainda dados de duas unidades de cuidados paliativos que mantêm-se na RNCCI do Alentejo por darem resposta a situações paliativas de complexidade baixa a moderada 21,22 .

\section{Aplicação inicial}

Foi feita uma observação inicial com aplicação do instrumento, nas duas primeiras semanas de fevereiro de 2018, a todos os doentes internados nas unidades da RNCCI do Alentejo. Foi dada formação aos profissionais de saúde sob o modo de preenchimento do instrumento através da disponibilização de um documento com as instruções de preenchimento.

\section{Tratamento e Análise dos dados}

Os dados foram recolhidos em suporte informático, através de um link disponibilizado a cada enfermeiro com responsabilidade na colheita da informação, e estes foram armazenados numa base de dados da ELCOS- Sociedade Portuguesa de Feridas.

Foi usado o teste de Shapiro-Wilk para avaliar a normalidade das variáveis numéricas e o teste de Levene para testar a homogeneidade das variâncias. O teste de 
Wilcoxon Mann-Whitney foi usado para avaliar se havia diferenças significativas no número de fatores de risco/antecedentes por sexo. Para medir a correlação entre o número de antecedentes/fatores de risco e a idade foi usado o coeficiente de correlação de Spearman. Para medir a correlação entre o número de antecedentes/fatores de risco e o número de feridas, bem com a relação entre a duração da ferida mais grave e a frequência do penso, foi usado o coeficiente de Kendall.

Para medir a correlação entre observadores foram avaliadas as discrepâncias nas respostas. A gravidade da discrepância foi classificada como leve ou grave. As discrepâncias nos fatores de risco, tipologia, origem e duração em anos das feridas foram consideradas graves porque têm implicações importantes nos resultados e consequentes intervenções. As discrepâncias nos antecedentes sociodemográficos, duração em dias e classificação das categorias das úlceras por pressão foram consideradas leves, porque não têm grandes implicações nos resultados e mesmo entre experts a categoria das UPP é um foco de discussão.

Para avaliar se havia relação entre a gravidade da ferida e o seu local de origem, e entre a localização da ferida mais grave e o número de intervenções nessa ferida, foi usado o teste do qui-quadrado de independência. Para avaliar se havia diferença significativa na duração da ferida pela sua gravidade foi usado o teste de Kruskal-Wallis.
Toda a análise estatística foi realizada com auxílio do software R, versão 4.0.3. O nível de significância considerado foi de $5 \%$.

\section{Aspetos éticos}

O presente estudo foi aprovado pela comissão de ética da Administração Regional de Saúde do Alentejo.

\section{RESULTADOS}

O instrumento final está disponível em: https://forms.gle/cZiWuFsqdMySm5te9 Fase 1

O instrumento final é constituído por quatro partes. A primeira parte recolhe variáveis sociodemográficas (local de registo do dados, idade e género do doente, estado civil, tipo de família, habilitações literárias e concelho de residência.

A segunda parte inclui as patologias associadas (aditiva; autoimune; cardíaca; cirúrgica; dermatológica; endocrinológica; gástrica; hematológica; neoplásica; neurológica; obstétrica / ginecológica; ortopédica; pediátrica / neonatológica; psiquiátrica; renal; respiratória; reumatológica; vascular e outra) e circunstâncias adversas (desnutrição; imobilidade; utilização de cadeira de rodas e outra).

A terceira parte do questionário recolhe a informação sobre a prevalência das diferentes tipologias de feridas (úlcera de perna venosa; úlcera de perna arterial; úlcera de perna de etiologia desconhecida; úlcera em 
pé diabético neuropático; úlcera pressão cat.1; úlcera pressão cat.2; úlcera pressão cat.3; úlcera pressão cat.4; úlcera pressão inclassificável; lesão por humidade; úlcera maligna; ferida cirúrgica; ferida traumática; queimadura; fístula; abcesso e outra). $\mathrm{O}$ registo da localização anatómica começa pela ferida mais grave (aquela que mais ameaça a vida, ou no caso de existirem várias feridas com a mesma gravidade aquela que apresenta maior profundidade) e permite registar até seis feridas por doente. Associado a cada ferida fica também registado o local de origem (domicílio; hospital; lar; unidade da rede nacional de cuidados continuados; unidade dos cuidados de saúde primários (UCSP)) e duração da mesma (em anos, meses e dias).

A quarta e última parte do instrumento, tem como objetivo recolher informação sobre o tempo de enfermagem dependido na realização do penso à ferida mais grave e frequência de realização do mesmo (opção de mais de uma vez/dia ou número de dias/ semana).

\section{Fase 2 e 3}

$\mathrm{O}$ instrumento foi alterado de acordo com as opiniões dos enfermeiros da prática. São aqui relatadas as principais alterações. No primeiro grupo de questões relativas ao perfil sociodemográfico: a área de residência foi substituída por concelho de residência habitual por apresentar maior clareza quanto ao que é pedido; na questão relativa aos antecedentes/fatores de risco passaram de 23 itens para 19 grupos de patologia, por existir inicialmente uma grande percentagem de respostas com "outra"; foi retirada a circunstância adversa "sedentarismo" por estar incluída na circunstância adversa imobilidade. No segundo grupo de questões relativas à nosologia das feridas, na tipologia das feridas foi acrescentada a opção "fistula"; foi retirada a opção de quem realiza o penso (enfermeiro ou cuidador informal) porque em todas as instituições avaliadas esta é uma responsabilidade do enfermeiro. A figura inicialmente presente para preenchimento da localização anatómica frequentemente não era preenchida, pelo que foi removida $\mathrm{e}$ substituída por opções com as diferentes localizações anatómicas.

O questionário inicialmente disponível em papel era de difícil preenchimento pelo número elevado de questões apresentadas numa folha única, pelo que passou a ficar disponível apenas em suporte informático.

\section{Fase 4}

Para medir a fidedignidade do instrumento, este foi aplicado por dois observadores numa amostra por conveniência de 16 utentes. Das 81 variáveis recolhidas no instrumento por dois enfermeiros, de forma independente, 65 (80\%) foram preenchidas de forma idêntica. Os pormenores das variáveis com pelo menos uma discrepância na colheita dos dados, estão apresentados na Tabela 1, em conjunto com o grau de discrepância por 
gravidade. Das 37 feridas observadas, três (8\%) tinham discrepâncias graves em pelo menos uma variável entre as duas observações, e 10 (27\%) tinham discrepâncias fracas em pelo menos uma variável.

Tabela 1 - Discrepâncias entre observadores

\begin{tabular}{|c|c|c|c|}
\hline Variável & Discrepância & Fraca & Grave \\
\hline Idade & 1 & $1(81 / 89)$ & \\
\hline $\begin{array}{l}\text { Habilitações } \\
\text { literárias }\end{array}$ & 1 & $1(<4,4$ anos $)$ & \\
\hline Patologias & 7 & & $\begin{array}{l}\text { 7: omissão de uma } \\
\text { comorbilidade }\end{array}$ \\
\hline $\begin{array}{l}\text { Circunstâncias } \\
\text { adversas }\end{array}$ & 1 & & $\begin{array}{l}\text { 1: omissão de uma } \\
\text { circunstância adversa }\end{array}$ \\
\hline Tipologia da ferida & 3 & $\begin{array}{l}2 \text { (cat } 3 / 4 \\
\text { UPP) }\end{array}$ & 1 (tipologia) \\
\hline Origem da ferida & 1 & & 1 (hospital/casa) \\
\hline Duração em anos & 1 & & $1(0 / 2)$ \\
\hline $\begin{array}{l}\text { Duração em } \\
\text { semanas }\end{array}$ & 1 & $1(0 / 1)$ & \\
\hline Tipologia da ferida & 1 & $\begin{array}{l}1 \text { (cat } 3 / 4 \\
\text { UPP) }\end{array}$ & \\
\hline $\begin{array}{l}\text { Duração em } \\
\text { semanas }\end{array}$ & 1 & $1(1 / 2)$ & \\
\hline Duração em dias & 1 & $1(2 / 4)$ & \\
\hline Tipologia da ferida & 2 & $\begin{array}{l}1 \text { (cat } 3 / 4 \\
\text { UPP) }\end{array}$ & 1 (tipologia) \\
\hline Localização & 2 & & $\begin{array}{l}1 \text { (localização) } \\
\text { 1: omissão de localização }\end{array}$ \\
\hline Tipologia & 2 & $\begin{array}{l}1 \text { (cat } 2 / 3 \\
\text { UPP) }\end{array}$ & 1 (tipologia) \\
\hline Localização & 1 & & 1(localização) \\
\hline
\end{tabular}

Fonte: Os autores.

\section{Aplicação inicial}

O instrumento foi aplicado em 138 dos 780 utentes internados nas UCCI da RNCCI do Alentejo, que apresentaram pelo menos uma ferida (prevalência 17,7\%). A prevalência foi mais elevada nas UMDR $(\mathrm{N}=65 / 231,28,1 \%)$ e menor nas ULDM $(\mathrm{N}=47 / 423,11,1 \%)$.

Um pouco mais de metade dos utentes (53\%) são do sexo feminino, com idades a variar entre os 40 e os 97 anos,sendo a idade mediana 83 anos (Tabela 2). Quase 4 em cada 5 utentes estão em ULDM ou UMDR.

Cerca de 3 em cada 4 utentes têm 2 a 5 patologias/circunstancias adversas (Tabela 2), sendo as mais usuais: hipertensão arterial $(62,3 \%), \operatorname{AVC}(36,2 \%)$, imobilidade $(36,2 \%)$, diabetes (29\%), dislipidemia $(23,9 \%)$ e insuficiência cardíaca $(21,0 \%)$. O número de fatores de risco não difere significativamente 
entre sexos $\left(\mathrm{Me}_{\mathrm{Masc}}=4 ; \mathrm{Me}_{\mathrm{Fem}}=4 ; \mathrm{p}=0,772\right)$ e $\left.\quad \mathrm{p}=0,432\right)$.

não está relacionado com a idade $\left(\mathrm{r}_{\mathrm{s}}=-0,068\right.$,

Tabela 2 - Características dos participantes com $\geq 1$ ferida, N (\%)

\begin{tabular}{|c|c|c|c|c|c|}
\hline Variável & & & $\begin{array}{c}\text { Feminino } \\
(N=73)\end{array}$ & $\begin{array}{c}\text { Masculino } \\
(\mathrm{N}=65)\end{array}$ & $\begin{array}{c}\text { Todos } \\
(\mathrm{N}=138)\end{array}$ \\
\hline \multirow[t]{7}{*}{ Idade } & Mediana (IIQ) & & $84(76-87)$ & $81(72-87)$ & $\begin{array}{c}83(75- \\
87)\end{array}$ \\
\hline & Categorias & $40-49$ & $2(2.7)$ & $2(3.1)$ & $4(2.9)$ \\
\hline & $\mathrm{N}(\%)$ & $50-59$ & $2(2.7)$ & $8(12.3)$ & $10(7.3)$ \\
\hline & & $60-69$ & $7(9.6)$ & $4(6.2)$ & $11(8.0)$ \\
\hline & & $70-79$ & $14(19.2)$ & $15(23.0)$ & $29(21.0)$ \\
\hline & & $80-89$ & $38(52.1)$ & $29(44.6)$ & $67(48.6)$ \\
\hline & & $\geq 90$ & $10(13.7)$ & $7(10.8)$ & $17(12.3)$ \\
\hline \multirow[t]{4}{*}{ Unidade } & $\mathrm{N}(\%)$ & UMDR & $40(54.8)$ & $25(38.5)$ & $65(47.1)$ \\
\hline & & ULDM & $23(31.5)$ & $24(36.9)$ & $47(34.1)$ \\
\hline & & UC & $10(13.7)$ & $13(20.0)$ & $23(16.7)$ \\
\hline & & UCP & $0(0)$ & $3(4.6)$ & $3(2.2)$ \\
\hline \multirow{5}{*}{$\begin{array}{l}\text { N. }{ }^{\circ} \text { de antecedentes } \\
/ \\
\text { fatores de risco }\end{array}$} & Mediana (IIQ) & & $4(3-5)$ & $4(2-5)$ & $4(2-5)$ \\
\hline & Categorias & $0-1$ & $5(6.9)$ & $4(6.2)$ & $9(6.5)$ \\
\hline & $\mathrm{N}(\%)$ & $2-3$ & $30(41.1)$ & $27(41.5)$ & $57(41.3)$ \\
\hline & & $4-5$ & $22(30.1)$ & $24(36.9)$ & $46(33.3)$ \\
\hline & & $\geq 6$ & $16(21.9)$ & $10(15.4)$ & $26(18.8)$ \\
\hline \multirow[t]{5}{*}{ N. ${ }^{o}$ de feridas } & Mediana (IIQ) & & $1(1-2)$ & $1(1-3)$ & $1(1-2)$ \\
\hline & Categorias & 1 & $39(53.4)$ & $35(53.9)$ & $74(53.6)$ \\
\hline & $\mathrm{N}(\%)$ & 2 & $21(28.8)$ & $13(20.0)$ & $34(24.6)$ \\
\hline & & 3 & $8(11.0)$ & $10(15.4)$ & $18(13.0)$ \\
\hline & & $\geq 4$ & $5(6.9)$ & $7(10.8)$ & $12(8.7)$ \\
\hline
\end{tabular}

IIQ = intervalo interquartil

Fonte: Os autores.

Menos de metade dos utentes tinham mais do que 1 ferida e quase 1 em cada 4 têm 2 feridas (Tabela 2). O número total de feridas por utente não difere entre sexos $\left(\mathrm{Me}_{\mathrm{Masc}}=1\right.$; $\left.\mathrm{Me}_{\mathrm{Fem}}=1 ; \mathrm{p}=0,701\right)$, e não está correlacionado com o número de antecedentes/fatores adversos $\left(\mathrm{r}_{\tau}=-0,013, \mathrm{p}=0,848\right)$. Não há diferença significativa na idade dos utentes por número de feridas $(\mathrm{p}=0,527)$.
No total foram indicadas 255 feridas distribuídas por mais de 60 localizações distintas. Das feridas registadas e que continham a sua localização (194), a maioria encontra-se nos membros inferiores (92\%). Não há diferença relevante na distribuição entre os sexos (Figura 1). 
Figura 1- Localização das feridas por gravidade

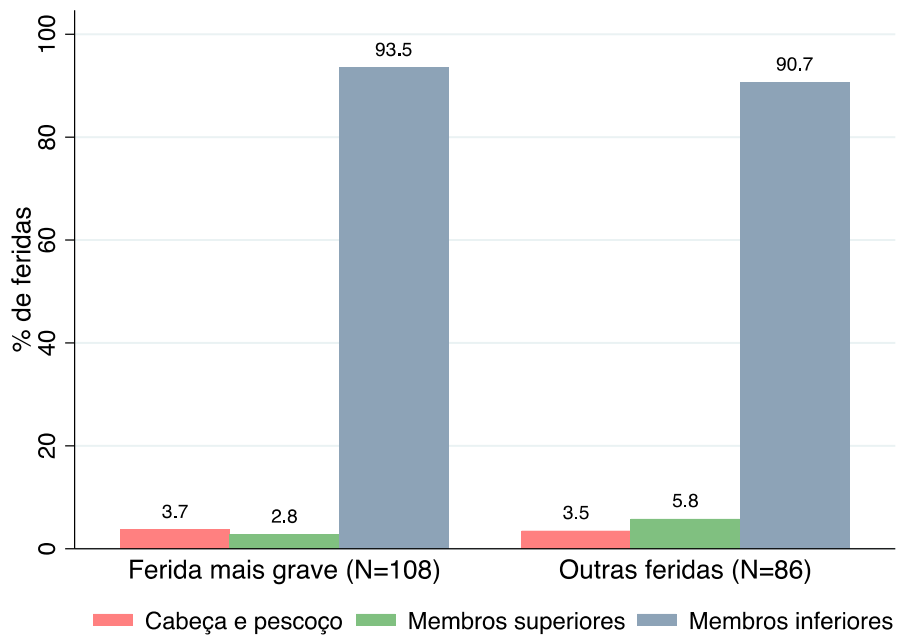

Fonte: Os autores.

O mais frequente é a ferida estar localizada na região sacrococcígea $(23,2 \%)$, calcâneo $(20,6 \%)$ e trocânter $(20,1 \%)$, sendo estas também as localizações mais frequentes da primeira e segunda ferida mais grave. As feridas com gravidade 3 ou superior estão quase todas localizadas no maléolo $(28,9 \%)$, no trocânter $(26,3 \%)$ e calcâneo $(23,7 \%)$.

Qualquer que seja a gravidade da ferida, a ferida mais comum é a úlcera por pressão de categoria 4 (Figura 2a). A segunda e terceira tipologias de ferida mais comuns variam com a gravidade da ferida, destacando-se as úlceras por pressão de categoria 3 , as feridas traumáticas, e as úlceras por pressão de categoria 1 e de categoria 2.

Figura 2 - Distribuição das feridas por a) tipologia e sexo, b) origem
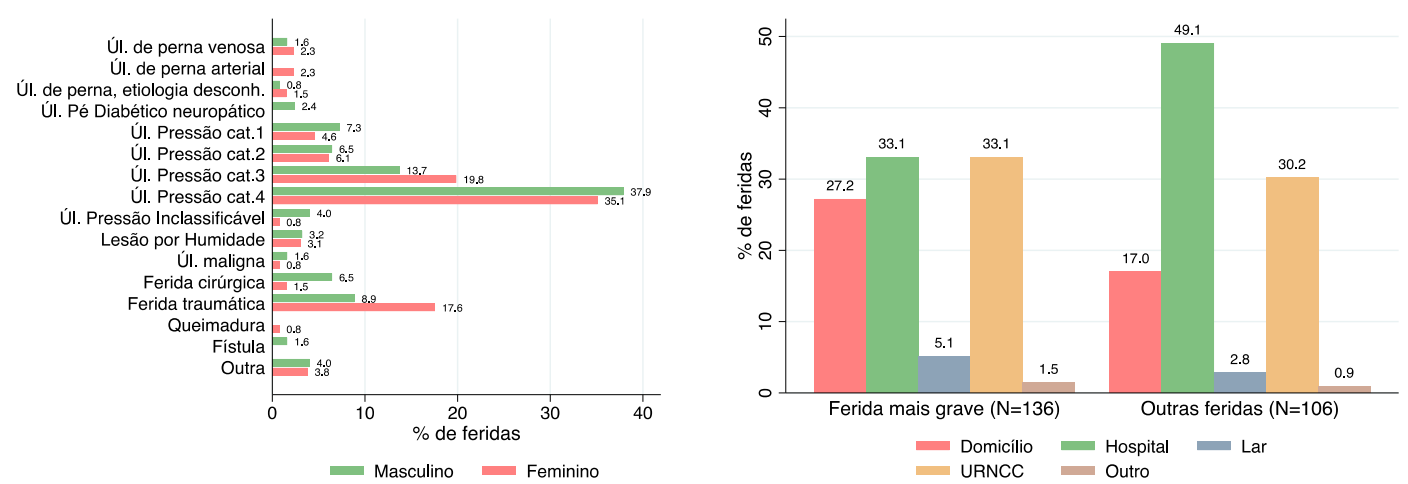

Fonte: Os autores. 
Predominam as que tiveram origem no hospital e a frequência aumenta à medida que reduz a gravidade da ferida (Figura 2b). Há relação significativa entre o grau de gravidade da ferida e o local esta teve origem $\left(\chi_{4}^{2}=\right.$ $13,698, \mathrm{p}=0,010)$, tal como seria de esperar, há mais feridas graves com origem no domicílio e menos com origem desconhecida.
A idade das feridas varia entre 1 dia e 30 anos, tendo 1 em cada 4 feridas menos de 1 mês e metade têm pelo menos 120 dias (Tabela 3). A idade das feridas não difere significativamente com a sua gravidade $\left(\chi_{4}^{2}=\right.$ $3,635, \mathrm{p}=0,458)$.

Tabela 3 - Duração da ferida e da realização do tratamento da ferida mais grave.

\begin{tabular}{llccc}
\hline Duração & & $\begin{array}{c}\text { Feminino } \\
(\mathbf{N}=73)\end{array}$ & $\begin{array}{c}\text { Masculino } \\
(\mathbf{N}=65)\end{array}$ & $\begin{array}{c}\text { Todos } \\
(\mathbf{N}=138)\end{array}$ \\
\hline $\begin{array}{l}\text { Das feridas } \\
\text { (dias) }\end{array}$ & Mediana (IIQ) & $195(36-300)$ & $84(28-240)$ & $120(30-270)$ \\
\cline { 2 - 5 } $\begin{array}{l}\text { Realização } \\
\text { do tratamento } \\
\text { (minutos) }\end{array}$ & Mediana (IIQ) & $13(10-2920$ & $1-11027$ & $1-11027$ \\
\cline { 2 - 5 } & Alcance & $2-60$ & $2-75$ & $15(10-30)$ \\
\cline { 2 - 5 } & & & & \\
\cline { 2 - 5 }
\end{tabular}

IIQ = intervalo interquartil

Fonte: Os autores.

O mais usual é o penso ser mudado 4 vezes por semana, seguindo-se 3 vezes por semana e diariamente (Figura 3a). A frequência de mudança do penso não revelou estar relacionado com a localização da ferida $\left(\chi_{4}^{2}=1,634, \mathrm{p}=0,803\right)$ nem correlacionado com a duração da ferida $\left(\mathrm{r}_{\tau}=0,052, \mathrm{p}=\right.$ 0,433).

A duração da realização do tratamento da ferida mais grave varia entre 2 e 75 minutos (Tabela 2) e há utentes com feridas muito antigas que continuam a mudar penso pelo menos uma vez por dia. O tempo que o penso demora a realizar difere significativamente com a localização da ferida $\left(\chi_{2}^{2}=9,870, p=0,007\right)$ (Figura 3b). As feridas localizadas na cabeça e pescoço demoram significativamente menos tempo a realizar o tratamento do que as feridas localizadas no tronco ou nos membros inferiores $(\mathrm{p}<0,05)$. 
Figura 3 - a) Frequência de mudança de penso da ferida mais grave; b) Duração da realização do tratamento em função da sua localização.

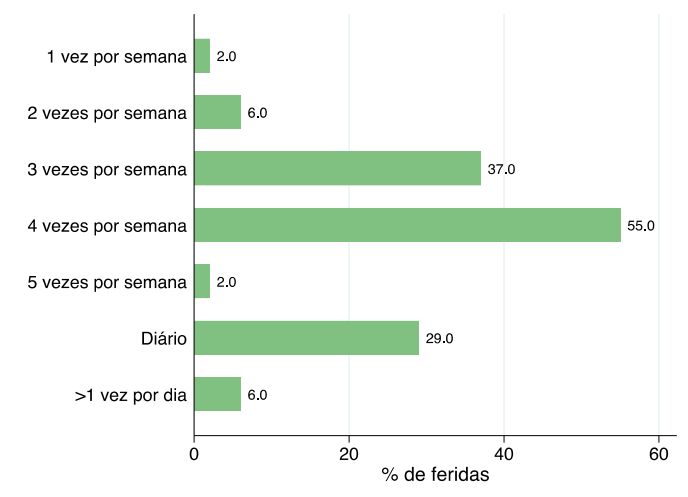

$\mathrm{a}$

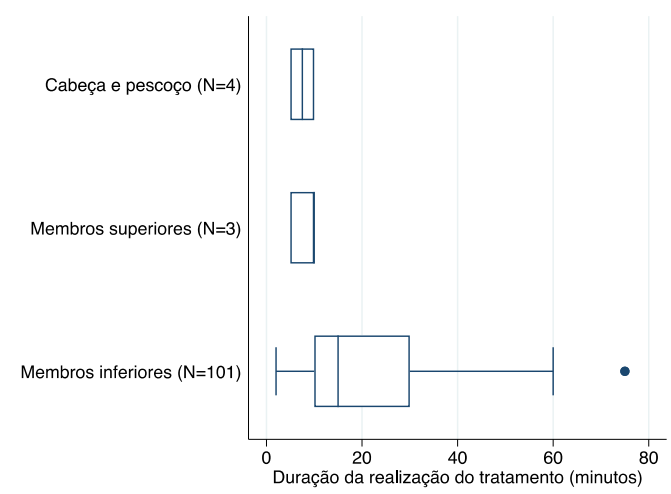

$\mathrm{b}$

Fonte: Os autores.

\section{DISCUSSÃO}

Os resultados obtidos na validação do presente instrumento indicaram níveis satisfatórios nas propriedades psicométricas avaliadas pelo que este pode ser utilizado com segurança na prática clínica, mediante a introdução de melhorias no manual de preenchimento. Os resultados da aplicação inicial do instrumento trazem dados, até agora inexistentes sobre a RNCCI. O tipo de feridas presentes, sua duração e cuidados associados , constituem importantes indicadores de desempenho, permitindo avaliar a efetividade e qualidade dos cuidados prestados ${ }^{15,23}$.

A prevalência de feridas encontrada é muito superior ao reportado em outros estudos em contextos ambientais semelhantes $2,4,24$. Contudo as metodologias apresentadas são muito variadas e muitos países não apresentam dados publicados.

A maioria das feridas encontradas neste estudo são úlceras por pressão categoria III e IV, o que é muito preocupante, dado que nas UCCI dentro e fora da Europa, a maioria das úlceras por pressão são de categoria I e II ${ }^{4,25}$. Este facto pode estar associado à presença de maior comorbilidades na população portuguesa ${ }^{6,14} 26$.

As discrepâncias entre observadores na classificação e etiologia das feridas encontradas neste estudo, evidencia a necessidade de fazer mais formação sobre a classificação das UPP ${ }^{27-29} 27$. O manual de instruções para preenchimento do instrumento deverá incluir imagens de feridas e respetiva classificação para ajudar o observador na tomada de decisão. Para aumentar a confiabilidade do instrumento sugere-se a aplicação do mesmo em diferentes contextos assistenciais.

Em futuras aplicações deste instrumento, é desejável que a observação direta dos doentes seja feita por dois observadores. A confiabilidade dos dados pode ser quantificada através de uma segunda visita surpresa efetuada por um enfermeiro 
perito a uma ou duas unidades escolhidas aleatoriamente ${ }^{30}$. Obviamente, esta medida implica o envolvimento de mais recursos humanos especializados.

Não obstante, a observação direta da pele dos doentes, através da aplicação deste instrumento, constitui uma avaliação mais fidedigna do que a recolha de informação retrospetiva com base em registos clínicos ${ }^{31}$. Nem sempre os profissionais de saúde estão despertos para a integridade cutânea dos seus doentes e nem sempre registam todas as feridas presentes.

Os resultados deste estudo irão ajudar na tomada de decisões nas UCCI, nomeadamente na distribuição de recursos materiais e humanos e na implementação de uma política de mudança que conduza a reduções na prevalência de feridas, em particular UPP ${ }^{32}$. Os recursos, até agora, alocados à educação, prestação de cuidados e investigação na área das feridas continuam a ser desproporcionalmente baixos e merecem uma atenção estratégica urgente ${ }^{33}$. O desafio nestes três domínios, educação, prestação de cuidados e investigação, é conseguir recrutar uma equipa multidisciplinar motivada para trabalhar, de forma coesa, em uníssono.

\section{Limitações do estudo}

Este instrumento não contém informação sobre as dimensões das feridas e recursos materiais associados ao seu tratamento, bem como se existe ou não infeção nas feridas identificadas. Este tipo de informação iria permitir estudar o impacto económico e estimar o tempo de cicatrização 34. As correlações foram analisadas sem considerar possíveis confounders.

Implicações para o avanço do conhecimento científico para a área de saúde e enfermagem

Existe um crescente interesse em fazer benchmarking internacional em termos da qualidade dos cuidados prestados nas UCCI e este instrumento construído e validado para a população portuguesa permite conhecer a dimensão do problema e obedece aos requisitos internacionais. As úlceras por pressão nas UCCI são um forte candidato para avaliações internacionais comparáveis.

\section{CONCLUSÃO}

Este instrumento, agora disponível, permite identificar o número e tipologia das feridas, caracterizar a gravidade, a localização anatómica, os cuidados associados ao tratamento e ainda identificar os antecedentes relevantes do doente.

Sendo as úlceras por pressão, o principal tipo de feridas identificado neste estudo, um problema complexo nas UCCI, a resolução passa igualmente por uma intervenção complexa junto dos profissionais e estruturas organizacionais. A forma como são implementadas as intervenções é fundamental e o contexto de cuidados não é só mais uma variável, mas um fator ímpar para o sucesso ou mudança de comportamentos. 
A falta de dados sobre feridas, comparáveis na Europa e a nível internacional nas UCCI, continuam a limitar o desempenho inteligente na tomada de decisão.

\section{REFERÊNCIAS}

1. Lanau Roig A, Fabrellas N, Saez Rubio G, Kate W. Time of chronic wound healing, as part of a prevalence and incidence study Tiempo de cicatrización de las heridas crónicas , a propósito de un estudio de prevalencia e. Enferm Glob. 2017;454-63.

2. Peckford S. Survey of Wound Prevalence in a Long-Term Care Facility. EWMA J. 2018;19(2):39-42.

3. Atkin L, Bucko Z, Conde Montero E, Cutting K, Moffatt C, Probst A, et al. Implementing TIMERS : the race against hard-to-heal wounds Inflammation / infection Social factors Edge Regeneration Moisture Tissue. J Wound Care. 2019;28(3):S1-50.

4. Moore Z, Avsar P, Conaty L, Moore $\mathrm{DH}$, Patton D, O'Connor T. The prevalence of pressure ulcers in Europe, what does the European data tell us: A systematic review. J Wound Care. 2019;28(11):710-9.

5. OCDE. Health at a Glance 2019 [Internet]. Organización para la Cooperación y el Desarrollo Económicos. 2019. 2-4 p. Available from: https://www.oecdilibrary.org/social-issues-migrationhealth/health-at-a-glance_19991312

6. Goes M, Lopes MJ, Oliveira $\mathrm{H}$, Fonseca C, Marôco J. A Nursing Care Intervention Model for Elderly People to Ascertain General Profiles of Functionality and Self Care Needs. Sci Rep. 2020;10(1):1-11.

7. Bonifant H, Holloway S. A review of the effects of ageing on skin integrity and wound healing. $\mathrm{Br} \mathrm{J}$ Community
Nurs. 2019;24(March):S28-33.

8. Parker CN, Finlayson KJ, Edwards HE, MacAndrew M. Exploring the prevalence and management of wounds for people with dementia in long-term care. Int Wound J. 2020;17(3):650-9.

9. Martinengo L, Olsson M, Bajpai R, Soljak M, Upton Z, Schmidtchen A, et al. Prevalence of chronic wounds in the general population: systematic review and meta-analysis of observational studies. Ann Epidemiol. 2019;29:8-15.

10. Poldrugovac M, Padget $\mathrm{M}$, Schoonhoven L, Thompson ND, Klazinga NS, Kringos DS. International comparison of pressure ulcer measures in long-term care facilities: Assessing the methodological robustness of 4 approaches to point prevalence measurement. $\mathbf{J}$ Tissue Viability [Internet]. 2021 Jan;(December 2020). Available from: https://doi.org/10.1016/j.jtv.2021.01.00 7

11. Pina E, Furtado K, Franks PJ, Moffat CJ.

Ulceras_de_Perna_em_Portugal_um_P roblema_de_Saude_.pdf.pdf. Rev Port Cir Cardio-Torácica e Vasc. 2004;XI(4):217-21.

12. Passadouro R, Sousa A, Santos C, Costa H, Craveiro I. Características e Prevalência em Cuidados de Saúde Primários das Feridas Crónicas. Rev da Soc Port Dermatologia e Venereol. 2016;74(November 2015):45-51.

13. Ministério da Saúde. Decreto de Lei 136/2015 de 28 de julho. Diário da República. Lisboa; 2015. p. 1-7.

14. ACSS-DRS. Relatório de monitorização da Rede Nacional de Cuidados Continuados Integrados (RNCCI). 2020.

15. Frijters DHM, Van Der Roest HG, Carpenter IGI, Finne-Soveri H, Henrard JC, Chetrit A, et al. The calculation of quality indicators for long term care facilities in 8 countries (SHELTER project). BMC Health Serv 
Res. 2013;13(1).

16. Edvardsson D, Baxter R, Corneliusson L, Anderson RA, Beeber A, Boas PV, et al. Advancing Long-Term Care Science Through Using Common Data Elements: Candidate Measures for Care Outcomes of Personhood, WellBeing, and Quality of Life. Gerontol Geriatr

Med. 2019;5:233372141984267.

17. Järbrink $\mathrm{K}$, Ni G, Sönnergren $\mathrm{H}$, Schmidtchen A, Pang C, Bajpai R, et al. Prevalence and incidence of chronic wounds and related complications: A protocol for a systematic review. Syst Rev. 2016;5(1):1-6.

18. Polit DF. Assessing measurement in health: Beyond reliability and validity. Int J Nurs Stud [Internet]. 2015;52(11):1746-53. Available from: http://dx.doi.org/10.1016/j.ijnurstu.201 5.07 .002

19. EPUAP Advisory Panel, NationalEuropen Pressure Ulcer Pressure Injuri Advisory Panel and Pan Pacific Alliance. Prevention and Treatment of Pressure Ulcers/Injuries: Quick Reference Guide. Emily Haesler (Ed.). EPUAP/NPIAP/PPPIA:2019. 2019.

20. Souza AC de, Alexandre NMC, Guirardello E de B. Propriedades psicométricas na avaliação de instrumentos: avaliação da confiabilidade e da validade. Epidemiol e Serv saude Rev do Sist Unico Saude do Bras. 2017;26(3):649-59.

21. Ministério da Saúde. Portaria $\mathrm{n}^{0} 75 / 2017.2017$;

22. Ministério da Saúde. Portaria n. ${ }^{0}$ 66/2018. Diário da República $\mathrm{n}^{\mathrm{o}}$ 46/2018, Série I de 2018-03-06 [Internet]. 2018;1177-82. Available from: https://dre.pt/home//dre/114822275/details/maximized

23. Edvardsson D, Baxter R, Corneliusson L, Anderson RA, Beeber A, Boas PV, et al. Advancing Long-Term Care Science Through Using Common Data
Elements: Candidate Measures for Care Outcomes of Personhood, WellBeing, and Quality of Life. Vol. 5, Gerontology and Geriatric Medicine. 2019. p. 233372141984267.

24. Anthony D, Alosoumi D, Safari R. Prevalence of pressure ulcers in longterm care: A global review. J Wound Care. 2019;28(11):702-9.

25. Commission CE. Pressure Injury Point Prevalence. 2019; Available from: https://tableau.albertahealthservices.ca/ \#/views/PressureInjuryPointPrevalence /PatientInformation?:iid=2

26. Gaspar de Matos S, Collier M, Marques A, Ferreira C, de Matos MG. Pressure ulcers: The challenge of monitoring in hospital context. Appl Nurs Res [Internet]. 2020;53(November 2019):151266. Available from: https://doi.org/10.1016/j.apnr.2020.151 266

27. Suva G, Sharma T, Campbell KE, Sibbald RG, An D, Woo K. Strategies to support pressure injury best practices by the inter-professional team: A systematic review. Int Wound $\mathrm{J}$. 2018;15(4):580-9.

28. Ahmajärvi KM, Isoherranen KM, Mäkelä A, Venermo M. A change in the prevalence and the etiological factors of chronic wounds in Helsinki metropolitan area during 2008-2016. Int Wound J. 2019;16(2):522-6.

29. National Pressure Ulcer Advisory Panel (U.S.), Al-Mansour LA, DudleyBrown S, Al-Shaikhi A, Chen G, Lin $\mathrm{L}$, et al. Organisation of health services for preventing and treating pressure ulcers. European Pressure Ulcer Advisory Panel and National Pressure Ulcer Advisory Panel PPPIA, editor. Int J Nurs Stud [Internet]. 2 nd editi. 2020 Mar 1 [cited 2016 Jul 22];29(1):1-9. Available from: http://www.epuap.org/wpcontent/uploads/2012/07/SCALEFinal-Version-2009.pdf 
30. Defloor $\mathrm{T}$, Clark $\mathrm{M}$, Witherow $\mathrm{A}$, Colin D, Lindholm C, Schoonhoven L, et al. EPUAP statement on prevalence and incidence monitoring of pressure ulcer occurrence. J Tissue Viability. 2005;15(3):20-7.

31. Defloor T, Schoonhoven L, Fletcher J, Furtado K, Heyman H, Lubbers M, et al. Statement of the European pressure ulcer advisory panel - Pressure ulcer classification. Differentiation between pressure ulcers and moisture lesions. J Wound, Ostomy Cont Nurs. 2005;32(5).

32. Pressure Ulcer Risk Assessment and Prevention: Comparative Effectiveness - Executive Summary | AHRQ Effective Health Care Program [Internet]. Available from: http://effectivehealthcare.ahrq.gov/inde x.cfm/search-for-guides-reviews-andreports/?pageaction=displayproduct $\& p$

roductid $=1490$

33. Demarré L, Van Lancker A, Van Hecke A, Verhaeghe S, Grypdonck M, Lemey $\mathrm{J}$, et al. The cost of prevention and treatment of pressure ulcers: A systematic review. Int $\mathbf{J}$ Nurs Stud [Internet]. 2015 Nov 1 [cited 2019 Sep 11];52(11):1754-74. Available from: https://www.sciencedirect.com/science/ article/abs/pii/S002074891500200X

34. Guest JF, Ayoub N, McIlwraith T, Uchegbu I, Gerrish A, Weidlich D, et al. Health economic burden that wounds impose on the National Health Service in the UK. BMJ Open. 2015;5(12):1-8.

Submissão: 2021-09-02

Aprovado: 2021-09-04 